\title{
SQUARE DETECTION BASED ON DISTANCE DISTRIBUTION
}

\author{
HONG-MIN LIU, ZHI-HENG WANG, CHAO DENG, LI-QIN JIA
}

\author{
School of Computer Science and Technique, Henan Polytechnic University, Jiaozuo 454000, China \\ E-MAIL: hongminliu@hpu.edu.cn, zhwang@nlpr.ia.ac.cn, super@hpu.edu.cn, lqjia@hpu.edu.cn
}

\begin{abstract}
:
This paper presents a method for the square detection based on distance distribution of the edge points of the image. The orientation line of the edge point is defined first, then for each pixel of the image, the distances between the pixel and the orientation lines of the edge points in the defined neighborhood of the pixel are computed to obtain the feature length and feature energy of the pixel, and further attain the feature length distribution map and feature energy distribution map of the whole image. Local maxima detection in the feature energy map is subsequently performed to find the potential center of the squares, and the corresponding feature length of the found potential center is also obtained. Additive steps are performed to verify and determine the centers of the squares. Synthetic images and natural images are used to prove the capability of the presented method for square detection. The experiments exhibit good performance for detecting the single square or the nested ones.
\end{abstract}

\section{Keywords:}

Square detection; Feature energy; Orientation line; Distance distribution

\section{Introduction}

Shape detection has been applied in various applications, such as automatic inspection, locating complex objects or tracking them, and assembly in industrial applications. Square is a common figure and is seen frequently in artificial scenes. The solution of the square detection can provide a general thought for the detection of the regular polygons.

The common methods for polygon detection are Hough transform (HT) based methods [1-3], which shown enough robust but along with the significant computation and large storage requirement. Other non-HT based methods were provided to avoid the defects of the HT method. Barnes et al. provided a robust regular polygon detection method[4-5], which derived the $\alpha$ posteriori probability for a mixture of regular polygons and thus the probability density function for the appearance of a set of regular polygons. Shi et al. [6] presented an automatically recognizing algorithm for geometrical shapes, which obtained the energy image and the directional angle image using the tunable filter from the original image, and then calculated the angle and the line number from the angle and perpendicular histogram to recognize the polygons. Siddharth and David [7] proposed an efficient method based on the matching. The method provided the polygon models assigned specific values of acuteness to each corner in a fixed-length sequence along the boundary in advance, then the geometrical similarity of the detected figures were compared with the models and specific polygons were detected. In addition, other methods were also provided for the polygons detection [8-9].

Compared with HT methods, non-HT methods have little needs for computation and storage, but these methods either aimed at specific polygons [4], or needed the information of the polygons [6-9], which are not universal for polygon detection. In this work, a general algorithm is presented for the square detection. The method is based on the distance distribution of the edge points. The method obtains the feature energy map and feature length map by computing the distance distribution of edge points for each pixel to determine the incenter and side length of the squares. This approach avoids the voting processing in HT-based algorithm, which raises the computation efficiency, and results in accurate detection of the arbitrary squares. The application results of the provided method to both synthetic and natural images are also presented.

The remainder of this paper is organized as follows. Section 2 introduces the principle of the method. Section 3 gives a detailed description for this method. Section 4 displays the experiment results on both synthetic and natural images. The conclusions are presented in Section 5.

\section{Principle of the method}

For the detection of a square, the orientation line of the edge points of the image is defined first. Wang et al. [10] presented the definition of the orientation line: For an edge point $P_{i}$, the line, which passed through the point and is vertical with the gradient detection, was defined as the 
orientation line of the point. As Fig.1 (a) shows, a unique inscribed circle of the arbitrary square is determined and the center is denoted as $c_{0}$. For edge point $P_{1}$, the orientation line of it is denoted as $l_{1}$ and displayed with red line in the figure. It can be seen that the orientation line of the edge point is coincident with the edge of the square, and the distance of the center $c_{0}$ to the orientation line $l_{1}$ is equal to the radius $r$ of inscribed circle.

For each pixel, compute the distances of the pixel to the orientation lines of edge points included in a defined area to obtain the distance distribution histogram of the pixel. Fig.2 (a) shows three different pixels. The neighborhoods of the pixels include different proportions of the square. Fig.2 (b) exhibits the distance distribution histogram of pixel A. The $x$-axis indicates the distance values of the point $A$ to the orientation lines of the edge points in the neighborhood, and $y$-axis counts the number of the edge points at some distance value. It can be seen that two edges of the square are included in the neighborhood of point A. Due to the definition of the orientation line, the distance values of the point $A$ to the orientation line of the edge points on one edge are equal, so only two distance values are obtained, as fig.2 (b) shows. For the pixel B, three edges are affected and so three peaks are displayed in its distance distribution histogram. The peak values reflect the contributions of different edges, as Fig. 2 (c) shows. When the pixel is the incenter of the square, the distances of the pixel to the orientation lines of the edge points are equidistant and equal to the radius of the inscribed circle, so only one distance value can be acquired. Fig. 2 (d) shows the distance distribution histogram of point $\mathrm{C}$, the incenter of the square, the distance value at which a maximum is got is the radius value of the inscribed circle, as well as one half of the side length of the square, and the maximum is approximately equal to the number of the edge points.

Consequently, due to the different positions of the pixels relative to the square, the distance distribution histogram of each pixel is different. This provides the new thought for the square detection.

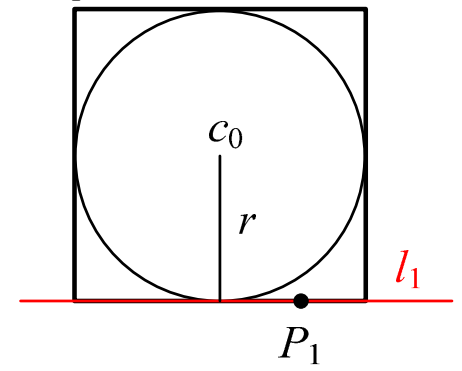

Figure 1 Character of the inscribed circle of the square

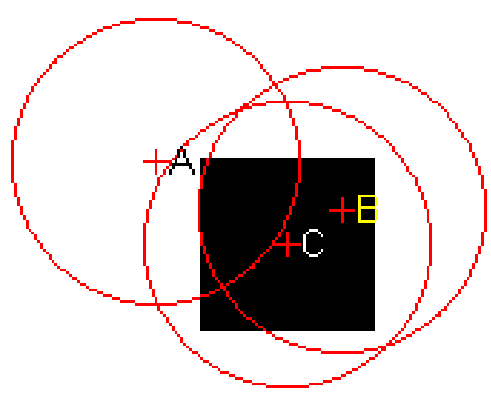

(a)

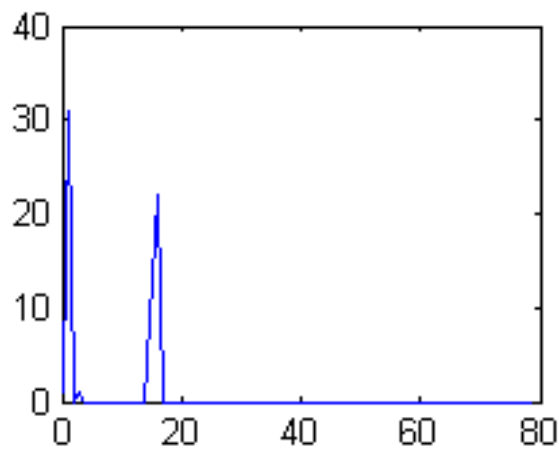

(b)

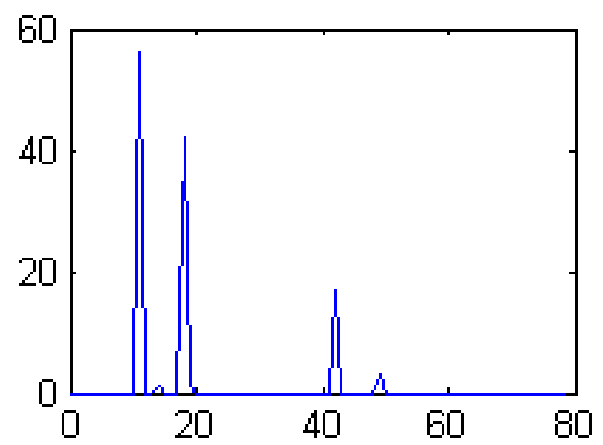

(c)

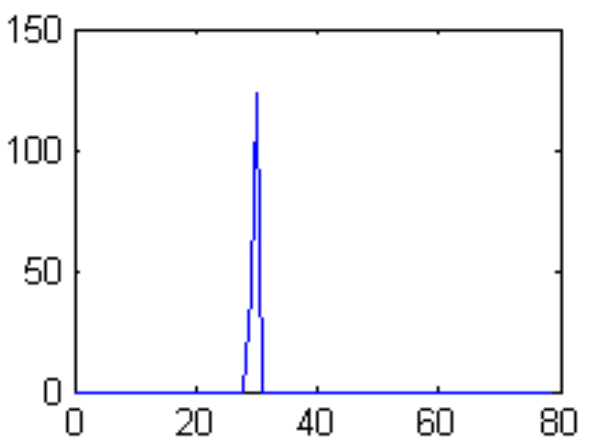

(d)

Figure 2 Distance distribution histograms of different pixels. (a) neiborhoods of appointed points relative to the square; (b) histogram of point A; (c) histogram of point B; (d) histogram of point $C$. 


\section{Algorithm}

Based on the principle expounded above, an algorithm is presented for detecting the squares. The steps are interpreted in the following.

\subsection{Computation of the orientation line of edge points}

For an edge point $P_{i}\left(x_{i}, y_{i}\right)(i=1,2, \ldots, N)$, assuming that $N$ edge points are obtained in the image, the gradient of the point $P_{i}\left(x_{i}, y_{i}\right)$ is computed and denoted as $\operatorname{grad}\left(X_{i}\right)=\left[d_{i x}, d_{i y}\right]$, in which $d_{i x}$ and $d_{i y}$ indicates the gradient along the $x$-axis and $y$-axis respectively. The orientation line of point $P_{i}\left(x_{i}, y_{i}\right)$ is defined as the line which gets across the point $P_{i}\left(x_{i}, y_{i}\right)$ and is perpendicular with the gradient vector of the point. The equation of the orientation line is denoted as $l_{i}: a_{i} x+b_{i} y+c_{i}=0$, in which $a_{i}=d_{i x}, \quad b_{i}=d_{i y}$ and $c_{i}=-d_{i x} x_{i}-d_{i y} y_{i}$. The orientation line of the edge point is coincident with the edge on which the point is located, as fig. 1 shows.

\subsection{Computation the feature length and feature energy}

Given an integer $L$, for a pixel $X(x, y)$, a neighborhood is defined as a circular area, which is centered on $X$ and of a radius of $L$. For each edge point $P_{i}(i=1,2, \ldots, N)$ in the neighborhood, the distance of the center pixel $X$ to the orientation line of the edge point $P_{i}$ is computed using the following equation:

$$
d_{i}=\left|a_{i} x+b_{i} y+c_{i}\right| / \sqrt{a_{i}^{2}+b_{i}^{2}}
$$

in which $a_{i}, b_{i}, c_{i}$ are the coefficients of the orientation line equation of edge point $P_{i}$. All the distances between the orientation lines of the edge points in the neighborhood and the center pixel $X$ are computed and the distance distribution histogram of the pixel $X$ is obtained. The maximum in the histogram is defined as the feature energy of pixel $X$ denoted as $E(x, y)$ and the corresponding distance is defined as the feature length of pixel $X$ denoted as $K(x, y)$. Compute the feature length and feature energy of each pixel to obtain the feature length distribution map and feature energy distribution map of the whole image

\subsection{Local maxima detection}

Local maxima detection is performed based on feature energy distribution map to find the potential incenter of the squares. Firstly, a threshold $T$ is applied to get rid of the most pixels. The threshold can be self-adapting using the mean and standard deviation of the feature energy distribution map. Then, the remained pixels are validated to determine the real incenter of the squares. For passing the validation, the ratio of $E(x, y)$ to $K(x, y)$ of the remained pixels should fulfill the following formula:

$$
E(x, y) / K(x, y)>(8 / \sqrt{2}) \cdot s
$$

As Section 2 says, $K(x, y)$ is equal to the half of the side length of a square, and $E(x, y)$ is approximately equal to the number of the edge points. With no rotation, the number of the edge points is equal to the perimeter of the square and the ratio of $E(x, y)$ to $K(x, y)$ is 8 . But with some rotation, the number of the edge points is not equal to the perimeter of the square, and the difference gets the maximum at $45^{\circ}$ rotation, where the ratio is obtained to be $(8 / \sqrt{2})$ in theory. For real images, a factor $s$ with a value of $0.5 \sim 0.7$ is multiplied. The pixels passing through the above two steps may correspond to the potential incenters of the squares, and the feature length of each incenter corresponds to the radius of the inscribed circle of a square.

\subsection{Detection of nested squares}

For nested squares, especially those sharing one center but with different radii of inscribed circles, a further detection is performed to validate if the incenter obtained is the same center of different inscribed circles of the squares. For that the feature energy and feature radius of each potential incenter is computed again with a difference compared with the method presented in section 3.2. In section 3.2, the feature energy is the maximum in the histogram of a pixel and the feature length is the distance corresponding to the maximum. Now in this step, the feature energy is a vector containing the local maxima in the distance distribution histogram of an incenter, the radii of potential concentric circles are the corresponding feature lengths. Then for each couple of feature energy and feature length, threshold and formula (2) are used again to validate the rationality of the couple. The feature length of remained couple is added as the radius of a new inscribed circle concentric with detected inscribed circle in section 3.3. 


\subsection{Detection of the squares}

For detecting the squares from other shapes, the gradient orientations of the edge points are used. When a center and radius of inscribed circle of a potential square is determined, the edge points are also obtained. For a square, there should be two main orientations in the gradient orientation distribution of the edge points. This restriction can be used to eliminate the non-square.

\section{Experiment results}

Experiments have been performed both on synthetic and natural images in order to evaluate the performance of the proposed method.

\subsection{Synthetic experiments}

As shown in figure 3 (a), a $398 \times 84$ pixel image including two squares with different rotation and a circle is generated. The feature energy distribution map of the synthetic image is shown in figure 3 (b), in which the intensities of potential incenters of the figures are larger obviously than other pixels. Fig.3 (c) shows the incenters and the inscribed circles of the figures. In the case of a circle, the inscribed circle can be considered as itself. The finally results are shown in fig. 3 (d), in which the squares are detected and the circle is rejected.

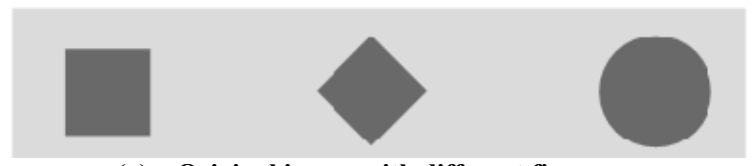

(a) Original image with different figures

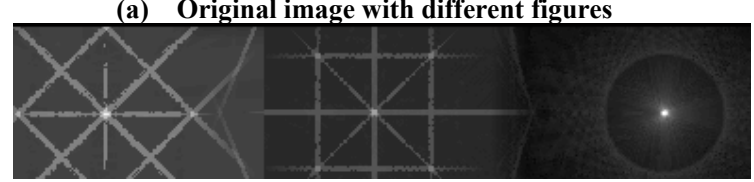

(b) Feature energy distribution map of the input image

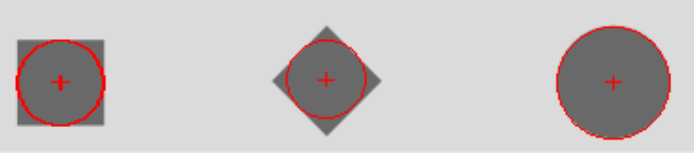

(c) Detected centers and inscribed circles of figures
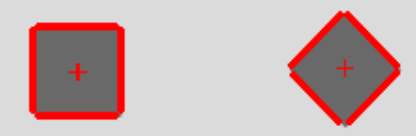

(d) Detected squares

Figure 3. Squares detection over synthetic image

Figure 4 shows the detection results of nested squares.
Three squares with different side lengths and rotational angles but the same center are presented. The feature energy distribution of the image is presented in Fig.4 (b), which is more complicated compared with the single square as shown in fig.3 (b). Fig.4 (c) displays the detected squares with edge points constructing the squares.

From these results, almost edge points of the square are detected except those around the corners of the square. The reason is mainly due to the lost of these points in the step of edge detection. In general, the proposed method shows good performance for the square detection.

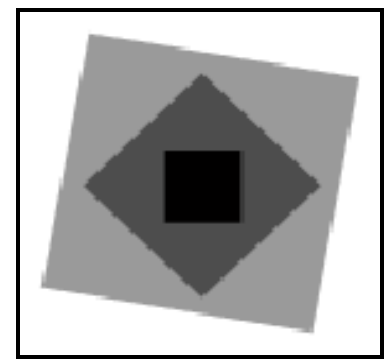

(a) Original image with nested squares

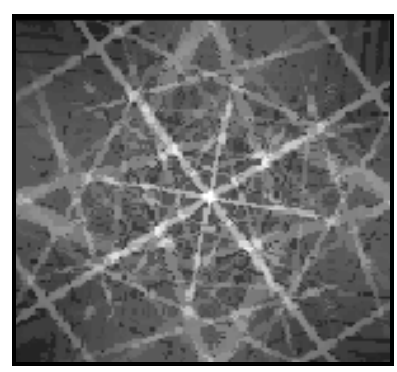

(b) Feature energy distribution of input image

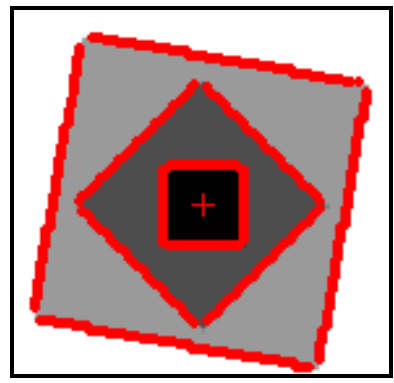

(c) Detected squares

Figure 4 Detection of nested squares

\subsection{Natural image experiment}

A natural image of $150 \times 166$ pixels is applied to test the performance of the presented method. The detected 
result is shown in Figure 5, in which the center of the foursquare outline of a watch is detected shown with "+" and the outline of the watch is shown with red edge points.

\section{Conclusions}

This paper has presented a method for automatically detecting the squares based on the distance distribution of the edge points. Distance distribution histograms of all pixels are computed to obtain the feature energy distribution and feature length distribution map of the image. The incenters of the squares are determined and the corresponding edge points are also obtained to display the detected squares. From our results, the proposed method shows good accuracy and consistency for the detection of the single square and the nested squares.

\section{Acknowledgements}

This work is supported by National Natural Science Foundation of China (61005033), Open Projects Program of National Laboratory of Pattern Recognition (20090018) and Doctor Degree Foundation of Henan Polytechnic University (B2010-39, B2010-68).

\section{References}

[1] Davies. E. R. Machine vision: theory, algorithms, practicalities (3rd Ed.). Elsevier, pp. 387-410, 2005.

[2] Chiu. S. H., Liaw. J. J. An effective voting method for circle detection. Pattern Recognition Letters, Vol 26, pp. 121-133, 2005.

[3] Torii. A., Imiya. A. The randomized-Hough -transform -based method for great-circle detection on

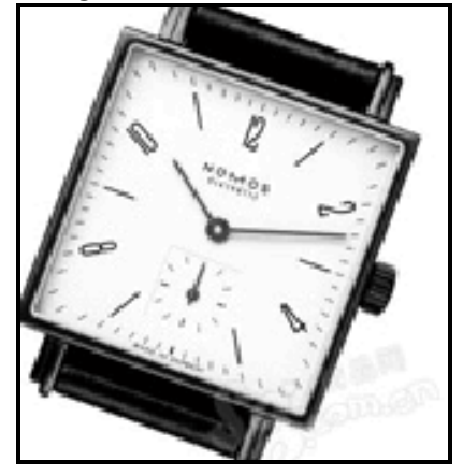

(a) A square watch sphere. Pattern Recognition Letters, Vol 28, pp. 1186-1192, 2007.

[4] Barnes N., Loy G. Real-time regular polygonal sign detection, Proceedings of the 5th International Conference on Field and Service Robotics (FSR05), pp. 55-66, 2005.

[5] Barnes N, Gareth. L., David. S. The regular polygon detector. Pattern Recognition, Vol 43, pp. 592-602, 2010.

[6] Shi. J., Xiao Z. H., Chang. Q. An Algorithm for Recognizing Geometrical Shapes Automatically Based on Tunable Filter. Journal of North University of China (Natural Science Edition), Vol 30, No. 5, pp. 467-471, 2009.

[7] Siddharth M., David W. P. Matching Flexible Polygons to Fields of Corners Extracted from Images. ICIAR 2007, Lecture Notes in Computer Science, 4633, pp. 447-459, 2007.

[8] Croitoru. A., Doytsher. Y. Right-angle rooftop polygon extraction in regularized urban areas: Cutting the corners. Photogrammetric Record, Vol 18, No. 108, pp. 311-341, 2004.

[9] Gates. J. W., Haseyama. M., Kitajima. H. Real-time polygon extraction from complex images. Circuits and Systems, 2000. Proceeding ISCAS 2000 Geneva. The 2000 IEEE Int'l Symp. on, Vol 5, pp. 309-312, 2000.

[10] Wang Z H, Wu F C, Hu Z Y. Corner Detection and Sub-Pixel Localization Based on Loeal Orientation Distribution. Journal of Software, Vol 19, No.11, pp. 2932-2942, 2008.

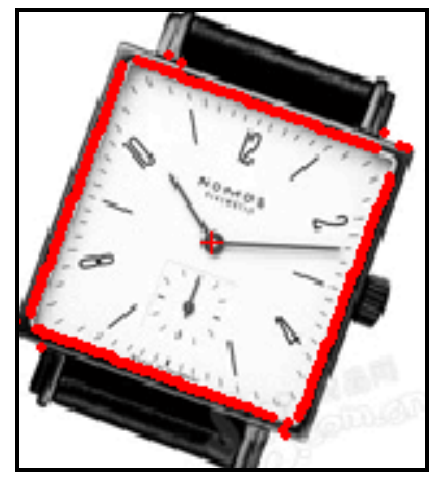

(b) Detected square

Figure 5. Square detection over natural image 